\title{
Transplanting a heart with a persistent left superior vena cava
}

\author{
Yaron D. Barac, MD, PhD, ${ }^{a}$ Dominick J. Megna, MD, ${ }^{\text {a }}$ Anthony W. Castleberry, MD, ${ }^{a}$ \\ Jacob N. Schroder, MD, ${ }^{a}$ Mani A. Daneshmand, MD, ${ }^{a}$ Joseph G. Rogers, MD, ${ }^{b}$ Chetan B. Patel, MD, \\ Mihai Podgoreanu, MD, ${ }^{\mathrm{c}}$ and Carmelo A. Milano, $\mathrm{MD},{ }^{\mathrm{a}}$ Durham, NC
}
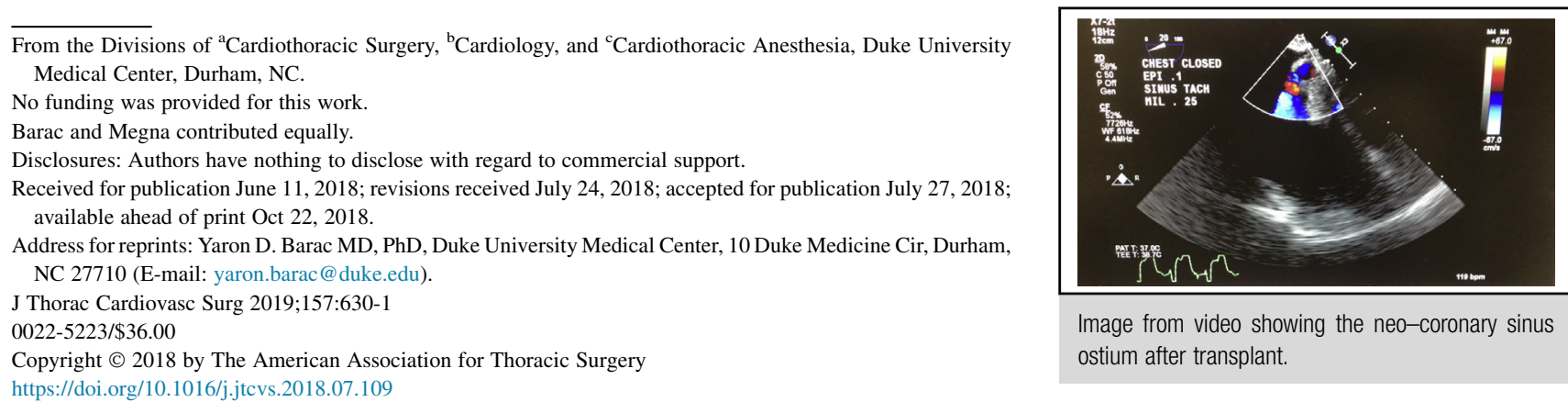

$\checkmark$ Video clip is available online.

\section{Central Message \\ We describe a suggested procurement sequence as well as cardiac transplantation modification when left superior vena cava is present.}

See Editorial Commentary page 632.
Left superior vena cava (LSVC) is the most common thoracic venous anomaly, with an occurrence of $0.3 \%$ to $0.5 \%$ in the general population. ${ }^{1}$ In $80 \%$ to $90 \%$ of cases, the LSVC drains into the coronary sinus; in the remainder, it drains into the left atrium, creating a right-to-left shunt. ${ }^{2}$ When considering using a heart with a known LSVC for transplantation, one should be aware that $40 \%$ of these hearts have associated anomalies, including coronary sinus ostial atresia, atrial septal defect, and bicuspid aortic valve, as well as conduction system abnormalities. ${ }^{3}$

LSVC in the donor heart may also impact preservation solution delivery during procurement. The LSVC may be connected to a coronary sinus with coronary sinus ostial atresia, meaning that the coronary sinus is actually draining into the LSVC, the innominate, the right superior vena cava, and then to the right atrium. Ligating the LSVC before delivery of the preservation solution thus may cause an injury and negatively affect posttransplant graft function. ${ }^{4}$

We chose to use a donor heart with LSVC; the donor was a 30-year-old man with blood type $\mathrm{O}$ who had died from gunshot trauma to the head. The LSVC was not identified or reported on donor chest computed tomography. The transthoracic echocardiogram demonstrated normal biventricular function, no valvular abnormalities, and no evidence of an LSVC. Intraoperative inspection of the donor heart revealed normal biventricular function and no palpable atherosclerotic coronary artery disease. Great vessel inspection demonstrated normal superior and inferior venae cavae, as well as an intact innominate vein. The LSVC was suspected during evaluation of the lungs and drawing of pulmonary venous blood samples. On elevation of the donor heart to identify the left pulmonary veins, an additional vascular structure was identified. A persistent LSVC was seen to be emptying into a dilated coronary sinus. This vessel resembled the pulmonary vein in appearance and was at first mistakenly identified as one of the left pulmonary veins. The LSVC was ligated; the aorta was then crossclamped, and the preservation solution was administered into the root.

At the implant operation, the donor heart was carefully inspected. The left sided SVC was probed and did not communicate with the right atrium. Inspection of the right atrium revealed absence of the ostium of the coronary sinus. A thin membrane separated the proximal coronary sinus from the right atrium; this membrane was incised to create a communication between the coronary sinus and the right atrium (Video 1).

A standard bicaval heart transplantation technique was used, and initial ventricular function in the recipient was normal. The chest was closed, and the patient returned to the intensive care unit. Within 24 hours, however, hemodynamics deteriorated and transesophageal echocardiography 


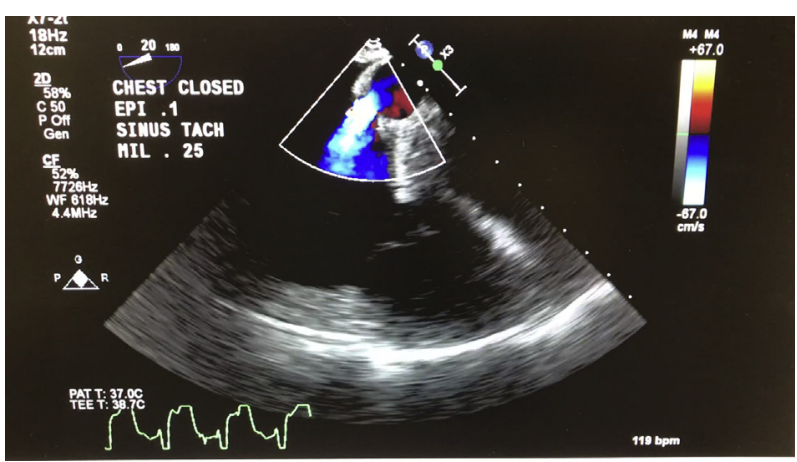

VIDEO 1. Video showing the neo-coronary sinus ostium after transplant. Video available at: https://www.jtcvs.org/article/S0022-5223(18)32472-3/ fulltext.

showed biventricular dysfunction with increased velocity across the opening from the coronary sinus to the right atrium.

The patient was returned to the operating room and placed on cardiopulmonary bypass. The right atrium was opened, the incision into the coronary sinus was extended several millimeters, and the opening was stretched with a surgical clamp. The patient was weaned from cardiopulmonary bypass, with echocardiographic resolution of the increased velocity at the coronary sinus-right atrium junction. Unfortunately, the patient required insertion of extracorporeal centrifugal ventricular assist devices, which was consistent with extensive procurement injury seen with routine endomyocardial biopsy. Slowly, during the course of days, left ventricular function improved, and the left ventricular assist device was weaned and removed. Unfortunately, the patient then had an acute intracranial hemorrhage, which progressed to herniation and brain death.

\section{TECHNIQUE}

Diagnosis of LSVC in the donor heart is possible with cardiovascular imaging (computed tomographic scan or echocardiography) or at the time of surgery. Increased concern should be raised by a deficient innominate vein or by an enlarged coronary sinus. If LSVC is identified, other anomalies should be excluded, and the transplant center and surgeon should decide whether any such anomalies disqualify the heart for transplantation.

It should be noted that the LSVC draining direction is unknown until the heart is procured and a coronary sinus ostium into the right atrium is identified. The procuring surgeon should assume that the coronary sinus drains into the LSVC and not directly into the right atrium; the LSVC should therefore not be ligated before delivery of the preservation solution.

After procurement, while preparing the heart for transplantation, the coronary sinus opening into the right atrium should be inspected. If no such opening is identified, an unroofing of the coronary sinus opening should be performed. A probe can be passed through the opening of the LSVC, and the blind end of the coronary sinus in the right atrium should be identified. A 1- to $2-\mathrm{cm}$ opening should be created; next, the LSVC should be oversewn. Failure to create an adequate communication between the coronary sinus and the right atrium may lead to increased coronary venous pressure and myocardial edema. This may also be manifested as increased velocity on echocardiography at the junction between the coronary sinus and the right atrium

\section{References}

1. Danielpour PJ, Aalberg JK, El-Ramey M, Sivina M, Wodnicki H. Persistent left superior vena cava: an incidental finding during central venous catheterization-a case report. Vasc Endovasc Surg. 2005;39:109-11.

2. Goyal SK, Punnam SR, Verma G, Ruberg FL. Persistent left superior vena cava: a case report and review of literature. Cardiovasc Ultrasound. 2008;6:50.

3. Voci P, Luzi G, Agati L. Diagnosis of persistent left superior vena cava by multiplane transesophageal echocardiography. Cardiologia. 1995;40:273-5.

4. Nicoara A, Ruffin D, Cooter M, Patel CB, Thompson A, Schroder JN, et al. Primary graft dysfunction after heart transplantation: incidence, trends, and associated risk factors. Am J Transplant. 2018;18:1461-70. 